\title{
The impact of the COVID-19 pandemic on an international rehabilitation study in MS: the CogEx experience
}

\author{
Anthony Feinstein ${ }^{1,23}$ (1) Maria Pia Amato ${ }^{2,3}$. Giampaolo Brichetto ${ }^{4,5}$. Jeremy Chataway 6,7 . \\ Nancy D. Chiaravalloti ${ }^{8,9} \cdot$ Gary Cutter $^{10}$. Ulrik Dalgas ${ }^{11}$. John DeLuca ${ }^{8,9} \cdot$ Rachel Farrell $^{6,7} \cdot$ Peter Feys $^{12}$. \\ Massimo Filippi ${ }^{13,14,15,16,17}$. Jennifer Freeman ${ }^{18} \cdot$ Matilde Inglese $^{19,20} \cdot$ Cecilia Meza $^{1} \cdot$ Rob Mot $^{21}$. \\ Maria Assunta Rocca ${ }^{13,14}$. Brian M. Sandroff ${ }^{8,9} \cdot$ Amber Salter ${ }^{22}$ on behalf of the CogEx Research Team
}

Received: 22 September 2021 / Revised: 27 October 2021 / Accepted: 28 October 2021 / Published online: 5 November 2021

(c) The Author(s), under exclusive licence to Springer-Verlag GmbH Germany 2021

\begin{abstract}
Pandemic restrictions have led to changes in therapy plans and disrupted rehabilitation services for people with multiple sclerosis. CogEx is an international, multicentre MS dual-intervention (cognitive rehabilitation, aerobic exercise) randomized, controlled rehabilitation trial confined to people with progressive disease. The primary outcome is cognition (processing speed).There are 11 treatment sites in six countries with participants required to make 27 site visits over 12 weeks. Collectively, the large, in-person demands of the trial, and the varying international policies for the containment of COVID-19, might disproportionately impact the administration of CogEx. During the first lockdown, all centres closed on average for 82.9 ( $\mathrm{SD}=24.3)$ days. One site was required to lockdown on two further occasions. One site remained closed for 16 months. Ten staff (19.2\%) were required to quarantine and eight staff (15.4\%) tested positive for COVID. 10 of 264 (3.8\%) participants acquired COVID-19. All survived. The mean duration of enrollment delay has been [236.7 ( $\mathrm{SD}=214.5)$ days]. Restarting participants whose interventions were interrupted by the pandemic meant recalculating the intervention prescriptions for these individuals. While the impact of the pandemic on CogEx has been considerable, all study sites are again open. Participants and staff have shown considerable flexibility and resilience in keeping a complex, international endeavour running. The future in general remains uncertain in the midst of a pandemic, but there is cautious optimism the study will be completed with sufficient sample size to robustly evaluate our hypothesis and provide meaningful results to the MS community on the impact of these interventions on people with progressive MS.

Trial registration: The trial was registered on September 20th 2018 at www.clinicaltrials.gov having identifier NCT03679468. Registration was performed before recruitment was initiated.
\end{abstract}

Keywords Multiple sclerosis $\cdot$ COVID-19 $\cdot$ Neurorehabilitation $\cdot$ Cognition $\cdot$ Exercise $\cdot$ Multisite $\cdot$ International

\section{Introduction}

To date, the SARS-CoV-2 has infected over 191 million people world-wide of whom 4,101,590 have died [1].There is no current evidence of people with multiple sclerosis (MS) having a higher rate of infection relative to the general population, but the pandemic restrictions have led to changes in therapy plans $[2,3]$ and disrupted rehabilitation services [4]. There are also data indicating that the pandemic has

Anthony Feinstein

ant.feinstein@utoronto.ca

Extended author information available on the last page of the article disrupted MS research that focuses on rehabilitation and quality of life [5].

CogEx ("Improving cognition in people with progressive MS: a multi-arm, randomized, blinded, sham-controlled trial of cognitive rehabilitation and aerobic exercise study") is an international, multicentre MS dual-intervention randomized, controlled rehabilitation trial funded by the MS Society of Canada. The current published cognitive rehabilitation literature in people with MS has many important limitations. With a few exceptions it is almost exclusively confined to individuals with relapsing-remitting disease. Sample size is typically small with studies lacking the statistical power to support the findings. Data generally come from a single centre raising questions about the broader applicability of the 
interventions. In many studies, impaired cognition is not a pre-requisite for study inclusion which can dilute the results of the chosen intervention. Furthermore, the overwhelming majority of studies entail a single intervention which means that the potential synergistic benefits of combined interventions have yet to be explored in a systematic way (see Deluca et al., [6] for a comprehensive review of the MS cognitive rehabilitation literature).

Being mindful of these limitations, the CogEx group of investigators designed a study that avoids these potential pitfalls. The methodology has been published elsewhere [7]. To summarize, the target sample size of 360 people is confined to people with progressive MS, making it the largest MS rehabilitation study in this group to date. There are 11 treatment sites across Canada, USA, England, Italy, Belgium, and Denmark. The principal investigators, all of whom have long track records of MS research, include neuropsychologists, neurologists, a psychiatrist, physiotherapists, exercise specialists, experts in neuroimaging, and statisticians. The study centres encompass general hospitals (with departments of neurology, psychiatry and rehabilitation medicine), and standalone rehabilitation institutes and neurological research and tertiary care centres.

The primary outcome measure is cognition, in particular information processing speed, with ambulation, memory, depression, and quality of life as secondary outcomes. A subset of participants undergoes both structural and functional MRI. Participants are randomized into four treatment arms, with biweekly interventions over 12 weeks: aerobic exercise (AE) and cognitive rehabilitation (CR); sham AE and CR; AE and sham CR; and sham AE and sham CR. With blinded assessments undertaken at baseline, 12 weeks and 6 months, participants are required to visit the treatment centre 27 times. The research assistants either administer the interventions or undertake the cognitive and motor assessments. No research assistant completes both tasks. This is to ensure that the testers are kept blind to group membership.

Collectively, the large, in-person demands of the CogEx trial, and the varying international policies for the containment of COVID-19, might disproportionately impact the administration of CogEx. Here, we report data on the impact of COVID-19 on CogEx participants, investigators, and staff.

\section{Method}

With the onset of the pandemic, CogEx sites began collecting data relating to number and duration of shutdowns, precautionary measures taken, infection rates in participants and investigators, quarantine rates, and COVID-related dropouts and cancellations.
Each study site obtained ethics permission for CogEx. Informed and signed consent were obtained from all CogEx participants.

\section{Results}

When the pandemic restrictions began in March 2020, $\operatorname{CogEx}$ was a third of the way through recruitment. The effects of the virus and the measures taken to counter it on the study over the past 16 months are shown in Table 1.

\section{Discussion}

The effects of the pandemic on CogEx have been significant, as demonstrated in the statistics in Table 1. There are, however, more subtle effects that are not shown (MS clinics shutting down and/or reopening to $50 \%$ or less capacity) and not known (how many potential participants did not respond to widely displayed flyers advertising the study) which have negatively affected recruitment. In addition, while the study was able to restart in 10 of 11 sites after a three-month shutdown, there was no anticipatory, pre-specified protocol in place for how best to resume the aerobic exercise and cognitive rehabilitation interventions in those participants whose treatment had been interrupted at different time points. Having been caught unaware by the pandemic, and with no precedent to guide our decisions for these specific interventions, we had to reach a decision on how best to restart the study.

To that end the following was decided by consensus amongst all the investigators: if participants were within the first 2 weeks of their interventions, they would begin the study anew. Participants within the last 2 weeks of their interventions would be deemed to have completed the study as they would have finished $80 \%$ of more of their treatments. For the remaining group, i.e. those participants in weeks three through nine inclusive, restarting would entail going back two sessions from their point of interruption. If this did not return subjects to where they had left off in terms of their CR and AE markers, additional make-up sessions were offered to reach these points. Once attained, these participants would complete the remaining weeks of the study. These same principles were applied to participants whose interventions were interrupted by the second and third lockdowns.

The challenges faced by CogEx are to varying degrees shared by other clinical trials that have been active during the pandemic $[8,9]$, although the nature and frequency of our interventions (24 face-to-face supervised treatment sessions in 12 weeks with pre and post assessments within this window along with longer-term follow-up) speaks to the particular intensity of our methodology. To date, the generic 
Table 1 COVID-19 related data

\begin{tabular}{|c|c|}
\hline Event & $\mathrm{N}(\%) /$ mean $(\mathrm{SD})$ \\
\hline Treatment centres closed during the first lockdown & $11(100 \%)$ \\
\hline Duration of first lockdown & 82.9 (24.3) days \\
\hline Treatment centres closed during second lockdown & $1 / 11(9.1 \%)$ \\
\hline Duration of second lockdown (applicable to one site) & 33 days \\
\hline Treatment centres closed during third lockdown & $1 / 11(9.1 \%)$ \\
\hline Duration of third lockdown (applicable to one site) & 64 days \\
\hline Treatment centres that have not yet reopened & $1(9.1 \%)$ \\
\hline Participants whose interventions were interrupted during first lockdown & 33 \\
\hline During weeks $1-3$ & 2 \\
\hline During weeks 4-9 & 21 \\
\hline During weeks $10-12$ & 10 \\
\hline Number of participants who contracted COVID (as of September 9, 2021) & $10 / 264(3.8 \%)^{*}$ \\
\hline Number of centres in which CogEx staff tested positive for COVID & $5 / 11(45.5 \%)$ \\
\hline Number of staff who tested positive for COVID & $8 / 52(15.4 \%)^{* *}$ \\
\hline Number of staff who had to quarantine because of exposure to COVID & $10 / 52(19.2 \%)$ \\
\hline $\begin{array}{l}\text { Number of centres reporting screened participants citing COVID concerns for dropping } \\
\text { out of the study }\end{array}$ & $11(100 \%)$ \\
\hline Number of screened participants citing COVID concerns for not enrolling in CogEx & $21 / 574(3.7 \%)$ \\
\hline Duration of enrollment delays & 236.7(214.5) days \\
\hline \multicolumn{2}{|l|}{ Precautions adopted $(n=10 \text { sites })^{* * *}$} \\
\hline Sanitize between participants & $10 / 10(100 \%)$ \\
\hline Masking & $10 / 10(100 \%)$ \\
\hline Symptom screening & $10 / 10(100 \%)$ \\
\hline Social distancing & $9 / 10(90 \%)$ \\
\hline Fewer treatment sessions on offer & $5 / 10(50 \%)$ \\
\hline Temperature check & $1 / 10(10 \%)$ \\
\hline
\end{tabular}

challenges faced by clinical trial lists during the pandemic have been addressed by the Food and Drug Administration (FDA) [10] and the Centers for Disease Control and Prevention (CDC) [11]. These guidelines were consulted, and where applicable followed, by the CogEx investigators as we devised our restart plans. A recent survey of 87 MS researchers from 18 countries revealed that almost $80 \%$ reported additional barriers to research during the pandemic, the two biggest being participant access and interruptions and delays in protocols. Female researchers perceived significantly more barriers compared to other genders [7].

CogEx is a demanding protocol for severely disabled people with MS [median Expanded Disability Status Score to date is 6.0 (IQR: 4.5, 6.5)] and keeping it running during a pandemic, also raised ethical questions. While all centres have been scrupulous in following government, provincial, local health authority and institutional mandates for continuing research, we needed to take into account the potential benefits of the interventions (which are unknown, hence the study) versus the risks to participants and staff of coming into medical institutions that in the majority of sites are also hospitals where COVID-19 patients were being treated. In weighing these competing factors, the investigators were aware that an important driving force behind CogEx is the dearth of rigorous, large scale empirical, multicentre rehabilitation data in people with progressive MS [12-14]. Furthermore, the study has been embraced by many of the participants who, historically as a group, have felt overlooked by MS researchers whose attention has focused predominantly on people with relapsing-remitting forms of the disease.

Despite the challenges summarized above, CogEx was able to reboot and at the time of writing has randomized 264 people to the four treatment arms. The core study design and interventions have not changed. To the best of our knowledge, no participant or investigator acquired COVID-19 from the study. The one site that remained closed for the past 16 months has just reopened. As we enter the last third of our study, we are close to being back to full strength in 
terms of personnel and clinics being fully opened. Looking to the future, we anticipate that the pandemic will have interrupted the interventions in approximately $10 \%$ of our sample. This group does not differ across the four treatment arms. The remaining $90 \%$ of participants will all have received their interventions without interruption and there will have been no delay in their timeline for the index-12week and 6-month assessments. The 21 screened participants who decided not to begin CogEx because of COVIDrelated worries had not yet been randomized.

The concerns with respect to the roughly $10 \%$ of participants whose interventions were interrupted by the pandemic are these: It is not known whether stopping and restarting the interventions after a delay will lessen the putative benefits of the interventions; how might the delayed timeline influence the outcome in individuals with a progressive disease course and how might being infected with COVID, even if the infection was mild, influence the response to the interventions. To address these questions, when it comes to analyzing the final data set, we will assess these potential confounders by analyzing our data with and without those participants who completed the modified protocol.

However, if we have learned one thing along this challenging journey, it is this: SARS-CoV-2 is here to stay. The vaccine rollout has some ways to go and new waves are predicted as the weather cools in the fall. How societies and medical institutions navigate this in the context of incomplete vaccinations will affect CogEx and our participants too. We are a large, multinational study and our composition is a plus, but during a pandemic, also a minus, given different regional approaches to managing this unprecedented health care challenge.

Faced with these uncertainties, it is helpful to recall what Robert Kennedy said in 1966 on a visit to the University of Cape Town: "Like it or not, we live in interesting times. They are times of danger and uncertainty; but they are also the most creative of any time in the history of mankind" [15]). The SARS-CoV-2 vaccine is proof of the latter. Our hope, is that CogEx will make a positive difference in the lives of people with progressive MS.

Funding Supported by the MS Society of Canada (Grant \#EGID3185).

Availability of data and materials To promote data transparency, anonymized data will be available upon reasonable request.

\section{Declarations}

Conflicts of interest Anthony Feinstein is on Advisory Boards for Akili Interactive and Roche, and reports grants from the MS Society of Canada, book royalties from Johns Hopkins University Press, Cambridge University Press, Amadeus Press and Glitterati Editions, and speaker's honoraria from Novartis, Biogen, Roche and Sanofi Genzyme. Maria Pia Amato received compensation for consulting services and/or speaking activities from Bayer, Biogen Idec, Merck-Serono, Novartis,
Roche, Sanofi Genzyme, and Teva Pharmaceutical Industries; and receives research support from Biogen Idec, Merck-Serono, Roche, Pharmaceutical Industries and Fondazione Italiana Sclerosi Multiplav. Giampaolo Brichetto has been awarded and receives research support from Roche, Fondazione Italiana Sclerosi Multipla, ARSEP, H2020 EU Call. Jeremy Chataway has received support from the Efficacy and Evaluation (EME) Programme, a Medical Research Council (MRC) and National Institute for Health Research (NIHR) partnership and the Health Technology Assessment (HTA) Programme (NIHR), the UK MS Society, the US National MS Society and the Rosetrees Trust. He is supported in part by the National Institute for Health Research, University College London Hospitals, Biomedical Research Centre, London, UK. He has been a local principal investigator for commercial trials funded by: Actelion, Biogen, Novartis and Roche; has received an investigator grant from Novartis; and has taken part in advisory boards/consultancy for Azadyne, Biogen, Celgene, MedDay, Merck and Roche. Nancy D. Chiaravalloti is on an Advisory Board for Akili Interactive and is a member of the Editorial Boards of Multiple Sclerosis Journal and Frontiers in NeuroTrauma. Ulrik Dalgas has received research support, travel grants, and/or teaching honorary from Biogen Idec, Merck-Serono, Novartis, Bayer Schering, and Sanofi Aventis as well as honoraria from serving on scientific advisory boards of Biogen Idec and Genzyme. John DeLuca is an Associate Editor of the Archives of Physical Medicine and Rehabilitation, and Neuropsychology Review; received compensation for consulting services and/ or speaking activities from Biogen Idec, Celgene, MedRhythms, and Novartis; and receives research support from Biogen Idec, National Multiple Sclerosis Society, Consortium of Multiple Sclerosis Centers, and National Institutes of Health. Cecilia Meza has no disclosures to report. Peter Feys is editorial board member of NNR and MSJ, provides consultancy to NeuroCompass and was board of advisory board meetings for BIOGEN. Massimo Filippi is Editor-in-Chief of the Journal of Neurology and Associate Editor of Human Brain Mapping, Neurological Sciences, and Radiology, received compensation for consulting services and/or speaking activities from Alexion, Almirall, Bayer, Biogen, Celgene, Eli Lilly, Genzyme, Merck-Serono, Novartis, Roche, Sanofi, Takeda, and Teva Pharmaceutical Industries, and receives research support from Biogen Idec, Merck-Serono, Novartis, Roche, Teva Pharmaceutical Industries, the Italian Ministry of Health, Fondazione Italiana Sclerosi Multipla, and ARiSLA (Fondazione Italiana di Ricerca per la SLA). Jennifer Freeman has been awarded research grants from the NIHR, UK. Matilde Inglese is Co-Editor for Controversies for Multiple Sclerosis Journal; received compensation for consulting services and/or speaking activities from Biogen Idec, Merck-Serono, Novartis, Roche, Sanofi Genzyme; and received research support from NIH, NMSS, the MS Society of Canada, the Italian Ministry of Health, Fondazione Italiana Sclerosi Multipla, H2020 EU Call. Robert W. Motl has no disclosures to report. Maria Assunta Rocca received speaker honoraria from Bayer, Biogen, Bristol Myers Squibb, Celgene, Genzyme, Merck-Serono, Novartis, Roche, and Teva and research support from the Canadian MS Society and Fondazione Italiana Sclerosi Multipla. Brian Sandroff has no disclosures to report. Gary Cutter is a member of Data and Safety Monitoring Boards for Astra-Zeneca, Avexis Pharmaceuticals, Biolinerx, Brainstorm Cell Therapeutics, Bristol Meyers Squibb/Celgene, CSL Behring, Galmed Pharmaceuticals, Horizon Pharmaceuticals, Hisun Pharmaceuticals, Mapi Pharmaceuticals LTD, Merck, Merck/Pfizer, Opko Biologics, OncoImmune, Neurim, Novartis, Ophazyme, Sanofi Aventis, Reata Pharmaceuticals, Teva pharmaceuticals, VielaBio Inc, Vivus, NHLBI (Protocol Review Committee), NICHD (OPRU oversight committee). $\mathrm{He}$ is on Consulting or Advisory Boards for Biodelivery Sciences International, Biogen, Click Therapeutics, Genzyme, Genentech, GW Pharmaceuticals, Klein-Buendel Incorporated, Medimmune, Medday, Neurogenesis LTD, Novartis, Osmotica Pharmaceuticals, Perception Neurosciences, Recursion/Cerexis Pharmaceuticals, Roche, TG Therapeutics. Dr. Cutter is employed by the University of Alabama 
at Birmingham and President of Pythagoras, Inc. a private consulting company located in Birmingham AL. Amber Salter is a statistical editor for Circulation: Cardiovascular Imaging.

Ethical standard statement The manuscript does not contain clinical studies or patient data.

\section{References}

1. COVID-19 Map_Johns Hopkins Coronavirus Resource Center. [cited 2021 Sep 17]. https://coronavirus.jhu.edu/map.html

2. Mateen FJ, Rezaei S, Alakel N, Gazdag B, Kumar AR, Vogel A (2020) Impact of COVID-19 on US and Canadian neurologists' therapeutic approach to multiple sclerosis: a survey of knowledge, attitudes, and practices. J Neurol 267:3467-3475. https://doi.org/ 10.1007/s00415-020-10045-9

3. Portaccio E, Fonderico M, Hemmer B, Derfuss T, Stankoff B, Selmaj K et al (2021) Impact of COVID-19 on multiple sclerosis care and management: Results from the European Committee for Treatment and Research in Multiple Sclerosis survey. Mult Scler J. https://doi.org/10.1177/1352458521100533910.1177/1352458521 1005339

4. Moss BP, Mahajan KR, Bermel RA, Hellisz K, Hua LH, Hudec T et al (2020) Multiple sclerosis management during the COVID-19 pandemic. Mult Scler J 26(10):1163-1171

5. Maguire R, Hynes S, Seebacher B, Block VJ, Zackowski KM, Jonsdottir J, et al (2021) Research interrupted: the impact of the COVID-19 pandemic on multiple sclerosis research in the field of rehabilitation and quality of life. Mult Scler J Exp Transl Clin 7(3):205521732110380. https://us.sagepub.com/en-us/nam/ open-access-at-sage

6. DeLuca J, Chiaravalloti ND, Sandroff BM (2020) Treatment and management of cognitive dysfunction in patients with multiple sclerosis. Nat Rev Neurol 16(6):319-32. https://pubmed.ncbi.nlm. nih.gov/32372033/

7. Feinstein A, Amato MP, Brichetto G, Chataway J, Chiaravalloti N, Dalgas U et al (2020) Study protocol: improving cognition in people with progressive multiple sclerosis: a multi-arm, randomized, blinded, sham-controlled trial of cognitive rehabilitation and aerobic exercise (COGEx). BMC Neurol. https://doi.org/10. 1186/s12883-020-01772-7
8. Lowe R, Barlow C, Lloyd B, Latchem-Hastings J, Poile V, Scoble $\mathrm{C}$ et al (2021) Lifestyle, Exercise and Activity Package for People living with Progressive Multiple Sclerosis (LEAP-MS): adaptions during the COVID-19 pandemic and remote delivery for improved efficiency. Trials 22(1):1-8. https://doi.org/10.1186/ s13063-021-05245-1

9. Weber MS, Nicholas JA, Yeaman MR (2021) Balancing potential benefits and risks of bruton tyrosine kinase inhibitor therapies in multiple sclerosis During the COVID-19 pandemic. Neurol Neuroimmunol Neuroinflamm. https://doi.org/10.1212/NXI.00000 00000001067

10. Turner JR (2020) New FDA guidance on general clinical trial conduct in the era of COVID-19 releasing guidances in final form key messages in the guidance on conduct of clinical trials of medical products during the COVID-19 pandemic. Ther Innov Regul Sci 54:723-724. https://doi.org/10.1007/s43441-020-00160-0

11. Healthcare workers: information on COVID-19|CDC. [cited 2021 Sep 17]. https://www.cdc.gov/coronavirus/2019-ncov/hcp/index. html

12. Feinstein A, Freeman J, Lo AC. Progressive multiple sclerosis 2 Treatment of progressive multiple sclerosis: what works, what does not, and what is needed. www.thelancet.com/neurology. 2015 [cited 2021 Jul 27]; www.thelancet.com/neurology

13. Fox RJ, Thompson A, Baker D, Brown D, Browne P, Chandraratna D et al (2012) Setting a research agenda for progressive multiple sclerosis: The International Collaborative on Progressive MS New Perspectives. Mult Scler J 18(11):1534-1540

14. Zackowski KM, Freeman J, Brichetto G, Centonze D, Dalgas U, DeLuca J et al (2021) Prioritizing progressive MS rehabilitation research: a call from the International Progressive MS Alliance. Mult Scler J 27(7):989-1001. https://doi.org/10.1177/1352458521 99997010.1177/1352458521999970

15. Kennedy RF. Day of affirmation address, University of Capetown, Capetown, South Africa, June 6, 1966 I JFK Library. Papers of Robert F. Kennedy. Senate Papers. Speeches and Press Releases, Box 2, “'Freedom \& Democracy' University of Cape Town, Cape Town, South Africa." John F. Kennedy Presidential Library. 1966 [cited 2021 Sep 17]. https://www.jfklibrary.org/learn/about-jfk/ the-kennedy-family/robert-f-kennedy/robert-f-kennedy-speec hes/day-of-affirmation-address-university-of-capetown-capet own-south-africa-june-6-1966

\section{Authors and Affiliations}

\section{Anthony Feinstein ${ }^{1,23}$ (1) Maria Pia Amato ${ }^{2,3} \cdot$ Giampaolo Brichetto $^{4,5}$. Jeremy Chataway ${ }^{6,7}$. Nancy D. Chiaravalloti ${ }^{8,9}$. Gary Cutter ${ }^{10}$. Ulrik Dalgas ${ }^{11}$. John DeLuca ${ }^{8,9} \cdot$ Rachel Farrell $^{6,7} \cdot$ Peter Feys $^{12}$. Massimo Filippi ${ }^{13,14,15,16,17}$. Jennifer Freeman ${ }^{18}$ - Matilde Inglese ${ }^{19,20} \cdot$ Cecilia Meza $^{1} \cdot$ Rob Mot $^{21}$. Maria Assunta Rocca ${ }^{13,14}$ - Brian M. Sandroff ${ }^{8,9} \cdot$ Amber Salter $^{22}$ on behalf of the CogEx Research Team}

1 Department of Psychiatry, University of Toronto and Sunnybrook Health Sciences Centre, Toronto, ON M5R 3B6, Canada

2 Department NEUROFARBA, Section Neurosciences, University of Florence, Florence, Italy

3 IRCCS Fondazione Don Carlo Gnocchi, Florence, Italy

4 Scientific Research Area, Italian Multiple Sclerosis Foundation (FISM), Via Operai 40, 16149 Genoa, Italy

5 AISM Rehabilitation Service, Italian Multiple Sclerosis Society, Genoa, Italy
6 Queen Square Multiple Sclerosis Centre, Department of Neuroinflammation, Faculty of Brain Sciences, UCL Queen Square Institute of Neurology, University College London, London WC1B 5EH, UK

7 National Institute for Health Research, University College London Hospitals, Biomedical Research Centre, London, UK

8 Kessler Foundation, East Hanover, NJ, USA

9 Department of Physical Medicine \& Rehabilitation, Rutgers New Jersey Medical School, Newark, NJ, USA 
10 Department of Biostatistics, University of Alabama at Birmingham, Birmingham, AL, USA

11 Exercise Biology, Department of Public Health, Aarhus University, Dalgas Avenue 4, 8000 Aarhus, Denmark

12 REVAL. Faculty of Rehabilitation Sciences, Hasselt University, Diepenbeek, Belgium

13 Neuroimaging Research Unit, Institute of Experimental Neurology, IRCSS San Raffaele Scientific Institute, Milan, Italy

14 Neurology Unit, IRCSS San Raffaele Scientific Institute, Milan, Italy

15 Neurorehabilitation Unit, IRCSS San Raffaele Scientific Institute, Milan, Italy

16 Neurophysiology Service, IRCSS San Raffaele Scientific Institute, Milan, Italy

17 Vita-Salute San Raffaele University, Milan, Italy
18 Faculty of Health, School of Health Professions, University of Plymouth, Devon, UK

19 Department of Neuroscience, Rehabilitation, Ophthalmology, Genetics, Maternal and Child Health, and Center of Excellence for Biomedical Research, University of Genoa, Genoa, Italy

20 Ospedale Policlinico San Martino-IRCCS, Genoa, Italy

21 Department of Physical Therapy, University of Alabama at Birmingham, Birmingham, AL, USA

22 Department of Neurology, Section on Statistical Planning and Analysis, UT Southwestern Medical Center, Dallas, TX, USA

23 Department of Psychiatry, Sunnybrook Research Institute, 2075 Bayview Avenue, FG 16, Toronto, ON M4N 3M5, Canada 